\title{
THE ROLE OF SERUM OSTEOPONTIN LEVELS IN EGYPTIAN WOMEN WITH POSTMENOPAUSAL OSTEOPOROSIS
}

\author{
Amira A. Atta* ${ }^{1}$, Fatma Alzahraa M. H. Ahmed ${ }^{2}$, Sameh ELZayat ${ }^{3}$, Hussein Al-Sawaf ${ }^{1}$ \\ ${ }^{1}$ Department of Biochemistry, Faculty of Pharmacy, Egyptian Russian University. \\ ${ }^{2}$ Department of Biochemistry, Faculty of Pharmacy (Girls), Al-Azhar University. \\ ${ }^{3}$ Department of Rheumatology, Faculty of Medicine (Boys), Al-Azhar University.
}

*Corresponding Author: Amira-ahmed@eru.edu.eg

\begin{abstract}
:
Background: Postmenopausal osteoporosis (PMO) is a debilitating disease induced by estrogen deficiency characterized by bone loss and bone micro-architecture degradation contributing to fragility fractures. Osteopontin (OPN) has been found to be involved in bone turnover by activating the resorption process.

Objectives: This study aim to evaluate the relationship between serum OPN levels and bone mineral density (BMD) for identification of female individuals at high risk of osteoporotic fractures.
\end{abstract}

Methods: A total of eighty eight postmenopausal women (PMW) were recruited in this study. Densitometry results addressed cases of the study into 3 groups: thirty with osteoporosis, thirty with osteopenia and twenty eight PMW without complications as a control group. Serum OPN was measured by using Enzyme-Linked immunosorbent assay (ELISA) kits and BMD was obtained at lumbar spine and femoral neck by dualenergy X-ray absorptiometry (DEXA).

Results: Osteopontin levels were significantly higher in osteoporotic group compared to osteopenic and control groups $(\mathrm{P}<0.001)$. Negative correlation was found between BMD and OPN in osteoporotic group. By using the Receiver Operating Characteristic (ROC) curve, the cutoff level of $\geq 10.294 \mathrm{ng} / \mathrm{mL}$ for OPN was chosen for suggesting osteoporosis which yield a sensitivity of $66.67 \%$ and specificity of $96.43 \%$. Also, there were a significant increase in serum ALP levels in osteoporotic and osteopenic groups compared to control group while serum $\mathrm{Ca}$ failed to show a significant difference between the studied groups. In addition, serum TAC showed a significant decrease in osteoporotic group compared to control group.

Conclusion: These findings suggest that the circulating OPN might play role in the pathophysiology of PMO. Its blood concentration can be used as a sensitive monitoring indicator for the early detection of osteoporosis independently of BMD.

Keywords: Bone turnover; Estrogen; Osteopontin; Osteoporosis. 


\section{Introduction}

Postmenopausal osteoporosis (PMO) is a silent, asymptomatic bone disease where bone resorption exceeds bone formation. It is characterized by decreased bone mass, disruption of bone microarchitecture and compromised bone strength, leading to fragility fractures. Estrogen deficiency increases the level of osteoclastic activity resulting in excessive bone resorption (Kanis et al., 2013).

Fragility fractures are a major clinical, social, economical and public health issue, yet identification of subjects at high risk of fracture remains challenging (Boonen \& Singer, 2008). Dual-energy X-ray absorptiometry (DEXA) is recognized as the dominant, simple tool used to quantify BMD. It is the most preferred technique used in clinical practice that can diagnose osteoporosis (Lorente Ramos et al., 2012).

However, there are several technical limitations in the general application of DEXA for diagnosis of PMO that must be recognized (Kanis et al., 2013). Observations have demonstrated that a majority of fragility fractures occur in subjects whose BMD values are not in the osteoporotic range, about 50\% of fractures associated with osteoporosis; subjects's T-score in the osteopenic range (Shetty et al., 2016).

A disease which can mimic osteoporosis, for example, osteomalacia. Osteoporosis is a debilitating disease of abnormally low bone mass, but, the existing bone is fully mineralized. The presence of osteomalacia, a common complication of poor nutrition. It is a disorder of mineralization, inadequate bone mineralization. Osteoid throughout the skeleton is incompletely calcified resulted in a large amount of un-mineralized osteoid and the bone is therefore "softened". DEXA will underestimate total bone matrix because of decreased mineralization of bone (Kanis et al., 2013). Another example for technical limitations of DEXA, osteoarthrosis at the spine or hip are common in elderly. Published clinical trials noted that, the presence of artifacts such as vertebral spurs, intervertebral bone bridges, subchondral bone sclerosis associated with degenerative joint diseases can often be detected on the scan and can lead to falsely elevated BMD making density measurements less reliable (Watts, 2004).

It has been concluded that, BMD measurements are not the optimal determinant factor for fracture risk in PMO (McClung, 2005) and must be correlated with serum levels of bone turnover markers (BTMs) which considered a mirror that reflect the status of bone remodeling (Kwon et al., 2018). The BTMs are classified into two categories depend on the metabolic phase during that they are produced, Bone Formation Markers and Bone Resorption Markers (Vasikaran et al., 2011).

In the literature, one new biochemical bone marker named Osteopontin has been associated with bone microstructure, independently of BMD. Published literature suggested that the circulating level of OPN was associated with an increased risk of incident fractures (Wei et al., 2016). Osteopontin is a secreted, multifunctional phosphoprotein, originally identified and isolated from the bone. It is a prominent constituent of the non-collagenous bone matrix; it is an extracellular matrix protein, secreted by both osteoblasts and osteoclasts, and is particularly concentrated at bone 
surfaces. It has been found in bone, the immune system, and the vascular system, being secreted in body fluids including milk, blood, and urine (Fodor et al., 2013).

Osteopontin has been shown to bind calcium ions, calcium oxalate crystal in urine and hydroxyapatite crystal (Chang et al., 2010). It suppresses the growth of calcium phosphate crystals (Boskey et al., 1993). Published studies have shown that OPN is an important regulator of inhibiting osteoblast proliferation and enhancing osteoclast differentiation (Franzén et al., 2008; Huang et al., 2004). In addition, OPN influences bone homeostasis by mediating attachment of osteoclasts to bone for bone resorption (Chang et al., 2010).

Therefore, it was found interesting to study the role of OPN as a diagnostic factor for early osteoporosis and to assess the relationship between OPN and BMD in postmenopausal Egyptian women.

\section{Materials and Methods}

This work was done at Al-Azhar University Hospitals during the period from November 2017 to November 2019. A total of eighty eight postmenopausal women in whom menstruation had been stopped for at least two year were consecutively enrolled for this study. The average age of the studied subjects was 56 years old ranging from 48 to 67 years old. All subjects were informed about the aim of the study, and then, a written informed consent for participation was obtained from each subject prior to any study-related procedures. The study protocol was approved by the Research Ethics Committee of the Faculty of Pharmacy (Girls), Al-Azhar University (approval number: 132, dated: October 2017).

\section{Case selection:}

Cases were selected from many patients came to the Rheumatology and Orthopedic out-patient clinics either seeking for medical advice because of bones and joints ache or referred from other centers for consultation, BMD determination and management. Of the initial 166 women, and after adequate general and clinical evaluation, 88 were considered eligible for the study. Observation of cases proved that none of cases had musculoskeletal deformities. None of the participants reported in their history that pain had interfered with daily activities. Also, none of the subjects reported a history of osteoporotic fractures and all were none smokers.

\section{General assessment and clinical evaluation:}

All cases were submitted to a structured scheduled protocol of assessment including a self-administered face-to-face validated questionnaire and interviews to allow for full history evaluation. Anthropometric measurements were also involved to collect data about body build and physical properties. Each of these women underwent the measurement of body weight, standing height, waist circumference, body mass index (BMI: weight/height ${ }^{2}\left[\mathrm{~kg} / \mathrm{m}^{2}\right]$ ), age of menarche and menopause. The patient's vital signs including blood pressure (BP), pulse and temperature were also recorded. 
Further information regarding identification of the subject state and to gather data on lifestyle, nutritional status, activities of daily living and physical performance.

In the present work, most of the enrolled cases practiced outdoor physical activity more than one hour daily.

A constant factor was considered for participation was the socio-economic state as it reflected the life style and nutritional status. The selected cases were more or less same standard, medium standard, none of the cases reported sedentary life

\section{Exclusion criteria:}

All subjects were reviewed clinically, all cases in addition were subjected to a reasonable specific laboratory studies to confirm general systemic fitness, to exclude chronic organs and systems illness and to isolate the appropriate cases for the study that satisfied the inclusion and exclusion criteria. Participants with any disease that could affect bone metabolism including diabetes mellitus, thyroid disorders, renal, pulmonary and hepatic diseases, malignant diseases, rheumatoid arthritis, or other metabolic diseases, all were excluded. In addition, we excluded subjects with history of hormonal therapy, chemotherapy and radiotherapy, as well as those taking medications that might interfere with bone metabolism such as corticosteroids, anticonvulsants, oral anticoagulants or therapy for osteoporosis, also women who were using supplements containing antioxidants, all were excluded.

\section{Serum collection and analysis:}

Appropriate blood sample was drawn into yellow gel vacutainer tubes from the antecubital vein in the morning after an overnight fasting. The samples were allowed to clot for 30 minutes at room temperature (RT) and then centrifuged at $3000 \mathrm{rpm}$ for 20 minutes. The serum was divided into two parts; one for immediate assay of calcium (Ca), alkaline phosphatase (ALP) and total antioxidant capacity (TAC). Other aliquoted sera were immediately stored at $-80^{\circ} \mathrm{C}$ until time of analysis of OPN.

Human enzyme-linked immunosorbent assay (ELISA) kit (Wuhan Fine Biotech Co., Ltd., Hubei, China) was used to measure serum OPN levels, using a quantitative technique called 'sandwich' technique. The serum concentration of $\mathrm{Ca}$, ALP and TAC were measured by colorimetric methods for the quantitative in vitro diagnostic measurement using kit manufactured by Bio-Diagnostics, Egypt. Samples from all women were analyzed at the same time of the day, and all assays were performed according to the manufacturer's instructions.

\section{Bone mineral density examination:}

Bone mineral density values for all candidates were measured with DEXA scanner at lumbar spine 1-4 (LS-BMD) and left femoral neck (FN-BMD). For all cases during the study, densitometry tests were performed by the same center and same system machine: GE Medical System (GE-Lunar Prodigy Primo) Madison, WI, USA Brand: GE-Lunar, Model: Prodigy Primo, SN: 35156. All BMD results were 
expressed as $\mathrm{g} / \mathrm{cm}^{2}$. According to world health organization (WHO) criteria for diagnosis and definition of osteoporosis, the studied cases were classified into 3 groups; normal with $\mathrm{T}$-score $\geq-1$, osteopenia with $-1<\mathrm{T}$-score $<-2.5$ and osteoporosis with $\mathrm{T}$ score $\leq-2.5$ (Kanis \& Kanis, 1994).

\section{Statistical analysis:}

Data were presented as mean \pm standard deviation (mean \pm SD). Testing for normality was done using Kolmogrov-Smirnov test and Shapiro-Wilk test, as the results showed that the data was normally distributed. A one-way analysis of variance (ANOVA) followed by Tukey's post-test to show any significant difference between individual groups. The statistical significance was set at $p$-value $<0.05$. Bivariate correlation analysis with Pearson correlation coefficient were performed to determine the relationship between serum OPN levels and BMD. By using the (ROC) curve, the cut-off values for serum OPN in classifying osteoporosis and osteopenia were determined. The Statistical Package for Social Sciences computer program (version 20.0 for Windows) was used for data analysis.

\section{Results}

In the study, BMD values based on WHO criteria classified subjects into 3 groups: 30 osteoporosis, 30 osteopenia, and the remaining 28 were apparently normal. The demographic, laboratory data and BMD values of the studied groups were listed in Table 1. Among the studied groups, no significant variation was found in mean value of age and BMI. The osteoporotic group had higher serum level of OPN compared to the osteopenic and control groups as illustrated in Figure 1. The BMD was significantly lower in osteoporotic PMW than those in osteopenic and control women. There were a significant increase in serum ALP levels in osteoporotic and osteopenic groups compared to control group while serum $\mathrm{Ca}$ failed to show a significant difference between the studied groups. In addition, serum TAC showed a significant decrease in osteoporotic group compared to control group.

To clarify the role of serum OPN in the PMO, we assessed the relationship between serum OPN levels and bone-related variables in patients and control women.

Table 2, showed that there was a significant negative correlation between serum OPN levels and DEXA parameters; LS BMD, LS BMD T-score, Lt. hip BMD and Lt. hip BMD T-score within osteoporotic patients, as illustrated in Figure 2.

We also assessed the relationship between TAC levels and DEXA parameters in the studied groups, the observations showed a significant positive correlation between TAC and LS-BMD and LS-BMD T-score in the osteoporotic group as shown in Table 3 and Figure 3.

As illustrated in Figure 4, the Receiver Operating Characteristic (ROC) curve analysis of OPN levels showed that the cutoff level of $\geq 10.294 \mathrm{ng} / \mathrm{mL}$ was chosen for suggesting osteoporosis, which yield an area under curve (AUC) equal to 0.909 (95\% CI $0.838-0.980 ; p<0.001$ ), a sensitivity of $66.67 \%$ and specificity of $96.43 \%$. OPN 
showed a sensitivity of $83.33 \%$ and specificity of $75 \%$ at a cutoff level of $\geq 7.352$ $\mathrm{ng} / \mathrm{mL}$ to suggest osteopenia which yielded an AUC equal to 0.819 (95\% CI 0.711 $0.927 ; \mathrm{p}<0.001)$.

Table 1. Clinical characteristics of the studied subjects.

\begin{tabular}{|c|c|c|c|c|}
\hline Parameters & Control & Osteopenia & Osteoporosis & p value \\
\hline Number & 28 & 30 & 30 & - \\
\hline Age (Years) & $54.4 \pm 3.7$ & $56.8 \pm 3.9$ & $58.7 \pm 4.7$ & $0.053^{\mathrm{a}}$ \\
\hline BMI $\left(\mathrm{kg} / \mathrm{m}^{2}\right)$ & $27.9 \pm 0.8$ & $28.1 \pm 1.1$ & $28.3 \pm 0.9$ & $0.275^{\mathrm{a}}$ \\
\hline $\operatorname{LS}$ BMD $\left(\mathrm{g} / \mathrm{cm}^{2}\right)$ & $1.08 \pm 0.12$ & $0.91 \pm 0.1 *$ & $0.8 \pm 0.07 *^{\#}$ & $<0.001^{\mathrm{a}}$ \\
\hline LS BMD T-score & $-0.13 \pm 0.66$ & $-1.76 \pm 0.43 *$ & $-3.23 \pm 0.57 * \#$ & $<0.001^{\mathrm{a}}$ \\
\hline FN-BMD $\left(\mathrm{g} / \mathrm{cm}^{2}\right)$ & $0.94 \pm 0.12$ & $0.82 \pm 0.11^{*}$ & $0.63 \pm 0.11 * \#$ & $<0.001^{\mathrm{a}}$ \\
\hline FN-BMD T-score & $-0.15 \pm 0.75$ & $-1.6 \pm 0.38^{*}$ & $-3.2 \pm 0.6^{*} \#$ & $<0.001^{\mathrm{a}}$ \\
\hline OPN (ng/mL) & $5.86 \pm 2.74$ & $9.48 \pm 2.68 *$ & $12.08 \pm 4.04 *^{\#}$ & $<0.001^{\mathrm{a}}$ \\
\hline $\mathrm{Ca}(\mathrm{mg} / \mathrm{dL})$ & $9.3 \pm 0.58$ & $9.2 \pm 0.61$ & $9.4 \pm 0.53$ & $0.457^{\mathrm{a}}$ \\
\hline ALP (U/L) & $90.7 \pm 13.8$ & $99.5 \pm 12.9 *$ & $100.1 \pm 13.4^{*}$ & $0.015^{\mathrm{a}}$ \\
\hline TAC (mM/L) & $1.26 \pm 0.39$ & $1.01 \pm 0.46$ & $0.91 \pm 0.36^{*}$ & $0.005^{\mathrm{a}}$ \\
\hline
\end{tabular}

Values were presented in terms of (mean \pm SD). BMI, body mass index; LS, lumbar spine; FN, femoral neck; BMD, bone mineral density; OPN, osteopontin; $\mathrm{Ca}$, calcium; ALP, alkaline phosphatase; TAC, total antioxidant capacity. ${ }^{\mathrm{a}} \mathrm{p}$ value by ANOVA. $* p<0.05$, significant from control group. \#p $<0.05$, significant from osteopenic group.

Table 2. Pearson correlation coefficients between serum OPN levels to other parameters in control, osteopenic and osteoporotic groups.

\begin{tabular}{|c|c|c|c|c|c|c|}
\hline \multirow[b]{3}{*}{ Parameters } & \multicolumn{6}{|c|}{ OPN (ng/mL) } \\
\hline & \multicolumn{2}{|c|}{ Control } & \multicolumn{2}{|c|}{ Osteopenia } & \multicolumn{2}{|c|}{ Osteoporosis } \\
\hline & $\mathbf{r}$ & p-value & $\mathbf{r}$ & p-value & $\mathbf{r}$ & p-value \\
\hline Age (Years) & 0.187 & 0.340 & 0.137 & 0.470 & 0.352 & 0.056 \\
\hline BMI $\left(\mathrm{kg} / \mathrm{m}^{2}\right)$ & -0.146 & 0.459 & -0.294 & 0.115 & -0.309 & 0.097 \\
\hline LS BMD $\left(\mathrm{g} / \mathrm{cm}^{2}\right)$ & -0.144 & 0.466 & -0.445 & 0.014* & -0.645 & $<0.001 *$ \\
\hline LS BMD T-score & -0.144 & 0.466 & -0.447 & $0.013 *$ & -0.634 & $<0.001 *$ \\
\hline FN-BMD $\left(\mathrm{g} / \mathrm{cm}^{2}\right)$ & -0.167 & 0.396 & -0.220 & 0.243 & -0.526 & $0.003^{*}$ \\
\hline FN-BMD T-score & -0.184 & 0.348 & -0.201 & 0.288 & -0.513 & $0.004 *$ \\
\hline $\mathrm{Ca}(\mathrm{mg} / \mathrm{dL})$ & -0.145 & 0.462 & -0.284 & 0.129 & -0.239 & 0.204 \\
\hline ALP (U/L) & 0.153 & 0.437 & 0.168 & 0.374 & 0.097 & 0.610 \\
\hline
\end{tabular}

The table shows Pearson correlation coefficients ( $r$ ) in the three groups. *p $<0.05$ is statistically significant. BMI, body mass index; LS, lumbar spine; FN, femoral neck; BMD, bone mineral density; OPN, osteopontin; $\mathrm{Ca}$, calcium; ALP, alkaline phosphatase. 
Table 3. Pearson correlation coefficients between serum TAC levels and DEXA parameters in the studied groups.

\begin{tabular}{|l|c|c|c|c|c|c|}
\hline \multirow{2}{*}{ DEXA parameters } & \multicolumn{7}{|c|}{ TAC (mM/L) } \\
\cline { 2 - 7 } & \multicolumn{2}{|c|}{ Control } & \multicolumn{2}{c|}{ Osteopenia } & \multicolumn{2}{c|}{ Osteoporosis } \\
\cline { 2 - 7 } & $\mathbf{r}$ & $\mathbf{p}$-value & $\mathbf{r}$ & $\mathbf{p}$-value & $\mathbf{r}$ & p-value \\
\hline LS BMD $\left(\mathbf{g} / \mathbf{c m}^{2}\right)$ & 0.189 & 0.336 & 0.260 & 0.166 & 0.363 & $0.048^{*}$ \\
\hline LS BMD T-score & 0.191 & 0.329 & 0.246 & 0.190 & 0.369 & $0.045^{*}$ \\
\hline FN-BMD (g/cm $\left.{ }^{2}\right)$ & 0.263 & 0.177 & 0.179 & 0.343 & 0.175 & 0.354 \\
\hline FN-BMD T-score & 0.269 & 0.166 & 0.170 & 0.369 & 0.164 & 0.387 \\
\hline
\end{tabular}

The table shows Pearson correlation coefficients (r) in the three groups. ${ }^{*} \mathrm{p}<0.05$ is statistically significant. LS, lumbar spine; FN, femoral neck; BMD, bone mineral density, TAC, total antioxidant capacity.

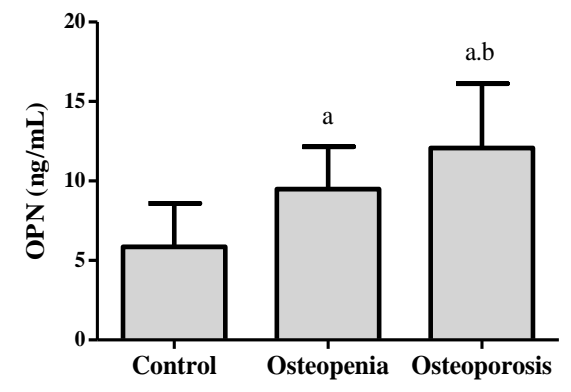

Figure 1. Bar chart of serum OPN levels of the studied groups. a: Significant from control group at $p<0.001$; b: Significant from osteopenic group at $p=0.007$.

(a)

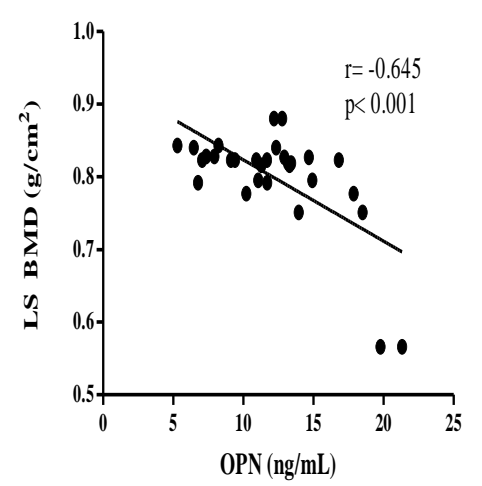

(b)

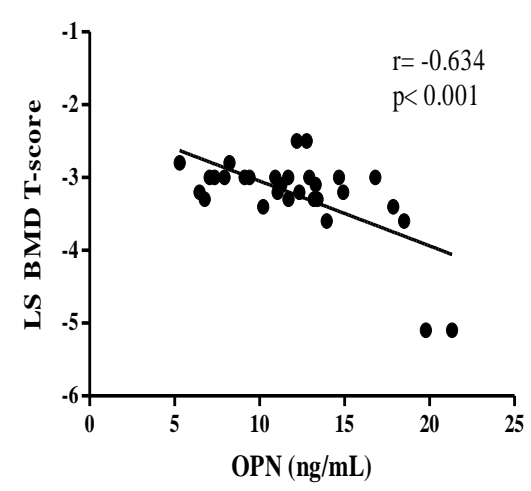


(c)

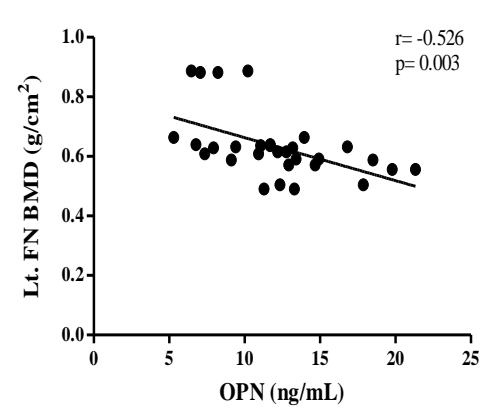

(d)

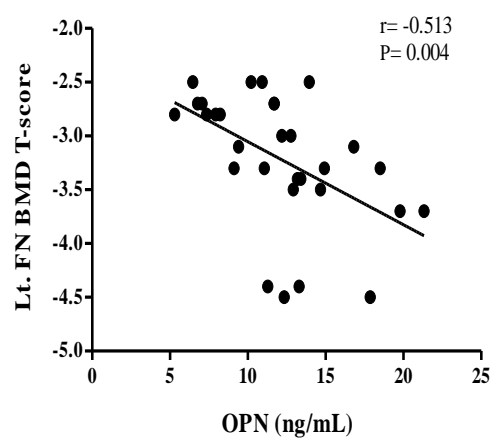

Figure 2. The Correlation of serum OPN and (a) LS BMD, (b) LS BMD T-score, (c) Lt. FN BMD, (d) Lt. FN BMD T-score in osteoporotic patients.

(a)

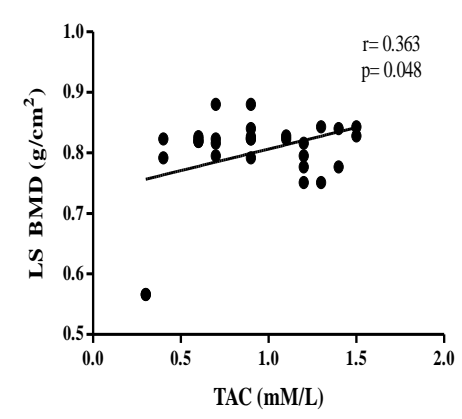

(b)

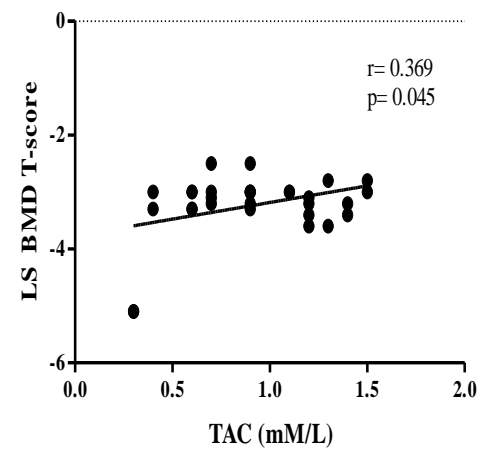

Figure 3. The Correlation of serum TAC and (a) LS BMD and (b) LS BMD T-score in osteoporotic patients.

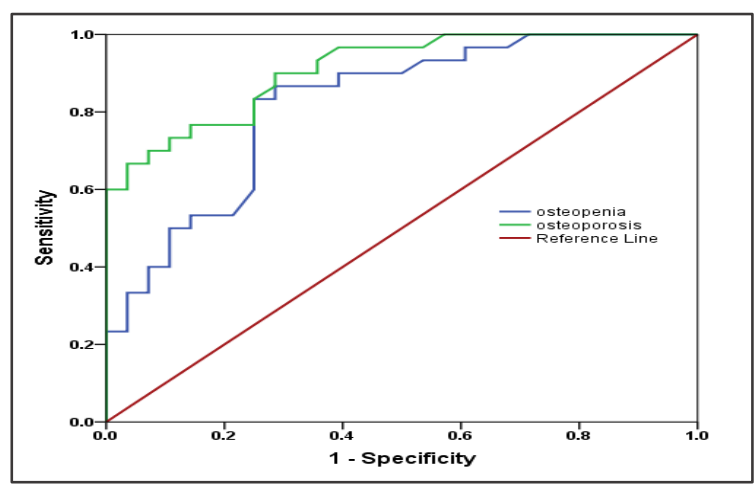

Figure 4. ROC curve for serum OPN. 


\section{Discussion}

Although for many years there was awareness of the morbidity and costs associated with osteoporotic fractures, a real progress can be achieved only through the early detection of osteoporosis before any fragility fractures occur (Blake, 2010).

DEXA is commonly used for diagnosis of osteoporosis by measuring BMD, but it has its technical limitations (Shetty et al., 2016). Moreover, BMD only assesses the mineral content in bones, which is only one component of bone strength (Chang et al., 2010). DEXA scanning can not define other factors contribute to fracture risk such as differences in bone material composition and structural design, both characteristics affect bone quality (Syed \& Khan, 2002). Also, BMD values do not detect the metabolic rate of bone turnover and do not define the molecular and cellular biology of bone (Kwon et al., 2018).

The biology of bone turnover can be assessed by BTMs. They are metabolic indicators released into serum and their quantity reflects the metabolic activity of bones. Markers of bone remodeling predominantly resorption markers have been shown to predict the risk for fractures, independent from BMD (Seibel \& Meier, 2010).

Osteopontin is non-collagenous bone matrix protein marker that implicated in bone metabolism and bone resorption in the remodeling process. In the current study, we demonstrated that group-III of osteoporotic PMW had a significant higher level of serum OPN compared to group-II with osteopenia and group-I of normal BMD, this was consistent with results obtained previously (Abdu Allah et al., 2017; Fodor et al., 2013; Wei et al., 2016). Wei et al (2016) stated that, this increased of serum OPN levels appear possibly as a concomitant event in high bone turnover state, which can explained by the fact that OPN enhances the expression of CD44 that causes an increase in osteoclast mobility and bone resorption (Cho et al., 2013).

Furthermore, Fodor et al (2013) stated that OPN bind to $\alpha_{v} \beta_{3}$ integrin of osteoclasts through RGD sequence, resulting in the activation of bone resorption and the decrease of bone mass. In addition, OPN expressed from osteoclasts is know to inhibit hydroxyapitate crytals formation, which indicate that OPN is associated with bone destruction and may be cosidered as the risk for bone fractures. Also, it acts as limiter limiting crystal formation in bones and teeth (Icer \& Gezmen-Karadag, 2018).

The current study demonstrated that serum OPN levels were inversely correlated with BMD of spine and hip in osteoporotic group. This was consistent with the findings of Wei et al., 2016 and Fodor et al., 2013 who indicated that serum OPN level has been shown to be an independent risk factor for low BMD in postmenopausal women and also can be used as a biomarker for early osteoporosis diagnosis.

The mean serum OPN levels in osteoporotic postmenopausal women recorded in the present study were $(12.08 \pm 4.04 \mathrm{ng} / \mathrm{mL})$ can be considered more or less matching with previous studies of Chang et al (2010) were (15.4 $\pm 6.2 \mathrm{ng} / \mathrm{mL})$, Wei et al (2016) were $(17.71 \pm 5.41 \mu \mathrm{g} / \mathrm{L})$ and Al-Najjar et al (2015) were $(16.41 \pm 6.33 \mathrm{ng} / \mathrm{mL})$, but 
was relatively low as compared to the published data by Cho et al (2013) were (43.6 \pm $25.9 \mathrm{ng} / \mathrm{mL})$.

The reason behind mean OPN differences between human studies not clear, but may be related to many factors such as the material used, the type of sample (plasma vs serum), age, race, detection method, and choice of ELISA kit (Cho et al., 2013). Chang et al (2010) suggested that, the cut off value of OPN should be dependent on the detection method.

In the present study, ROC curve analysis showed that a cutoff value of OPN in osteopenic group was $\geq 7.352 \mathrm{ng} / \mathrm{mL}$ and in osteoporotic group was $\geq 10.294 \mathrm{ng} / \mathrm{mL}$. These values are considered to be a threshold pre-diagnostic signs and a risk factor independently of age and BMD. Women that have serum OPN levels of $7.352 \mathrm{ng} / \mathrm{mL}$ or greater should be considered a patient more vulnerable for osteoporosis to occur and subsequent fracture risk.

After analysis study of 362 PMW done by Wei et al (2016), who concluded that the cutoff values of serum OPN for defining osteoporosis and osteopenia at the lumbar spine were $10.1 \mu \mathrm{g} / \mathrm{L}$ and $7.3 \mu \mathrm{g} / \mathrm{L}$ respectively. They indicated that PMW with serum OPN levels more than $7.3 \mu \mathrm{g} / \mathrm{L}$ should detect BMD at regular intervals to check whether osteoporosis or osteopenia happened. Also, Fodor et al (2013), suggested that serum OPN levels higher than $9.47 \mu \mathrm{g} / \mathrm{L}$ were the threshold for diagnosing osteoporosis at lumbar spine.

The relationship between age/body weight and osteoporosis is well known. The rate of osteoporosis increase with age (Chang et al., 2010). Obese PMW are considered protected against bone loss owing to endogenous estrogen production (Migliaccio et al., 2011). The study by Fodor et al (2013), suggested that, BMD varies markedly with age, because age contributes to risk independently of BMD. The same findings were reported by Al-Nejjar et al (2015) they stated that, aging means longer total exposure to chronic oxidant stress and inflammation, both of which contribute to development of osteoporosis.

Observation showed that most women achieved peak bone density by 18 to 25 years of age. After peak bone density is achieved, bone continues to be remodeled throughout a person's lifetime to respond to mechanical load and to replace older weakened bone. Estrogen deficiency caused by menopause, result in derangement of this homeostasis (Golob \& Laya, 2015). It is important to allude to the fact that, in menopausal women with age advancing, there is a doubled mechanism of bone loss; the rapid process is estrogen-deficiency bone loss occur at a rate of $2 \%$ to $3 \%$ of total bone mass per year, which begin after menopause and my last from 6 to 10 years. Subsequently, it is followed by a constant stage that is age-related bone loss (Chang et al., 2010).

Previous reports of Chang et al (2010), Fodor et al (2013) and Wei et al (2016), studied the relationship between serum OPN levels, age and body weight, they found a close association between them, but our study differs from the previous studies in that 
our data failed to show any correlation. Same findings were reported by Cho and his colleagues (Cho et al., 2013).

In the current study, the correlation between serum OPN levels and clinical parameters; age and BMI were studied. The obtained data failed to show any correlation. The possible reasons for lack of relationship in our study may be related to the number and the individual characters of the subjects included in the study. Anthropometric measurements showed no significant physical variations, a factor making our study limited to either within average weight or slight over-weight.

Gundberg (2000), stated that serum levels of ALP often increased in conditions associated with marked osteoblastic activity and bone formation. In the context of osteoporosis, he reported that, it may be modertately increased due to the generalized increased bone turnover to balance the remodeling processes in osteoporotic bone. In the current study, regarding ALP levels, none of the cases had a high serum level of ALP. However, observation showed a significant increase of ALP in osteoporotic and osteopenic groups when compared to control group. Similar findings were reported by Delmas et al (2000) that explained the reason of normal serum ALP levels in osteoporotic patients is that the serum pool of total ALP include several isozymes from different tissues such as liver, bone, spleen, intestine, kidneys and placenta.

The skeleton serves as a reservoir for more than $99 \%$ of the body's calcium supply, which is mobilized when serum calcium levels are low. Adequate calcium levels are crucial for bone health, clotting mechanisms and many other physiological functions (Veldurthy et al., 2016). The body systems including the skeletal, digestive system and kidney are worked together to maintain calcium homeostasis. The smallest drop in serum calcium below the normal level will trigger an immediate response. The body is ready to transfer calcium from other sources to maintain normal serum calcium levels and prevent hypocalcemia usually within minutes using one of these three systems (Beto, 2015).

Regarding serum calcium levels, the present study showed a normal range in all cases, as well as no significant difference between the studied groups. It is important to allude to the fact that the pathological defect in osteoporosis is quantitative not qualitative, osteoporosis is a debilitating disease of abnormally low bone mass, the existing bone is fully mineralized (Kanis et al., 2013). In addition, the statistical analysis showed no correlation between ALP, Ca, and OPN.

Oxidative stress (OS), can be defined as an imbalance in the human oxidative anti-oxidative status. Over-generation of free radicals and reactive oxygen species (ROS), which can adversely affect the integrity of various important biological molecules including; lipids, proteins, carbohydrates and DNA (Bergamini et al., 2004). In the female population, PMW are regarded as more vulnerable to OS than those in reproductive age, because their oxidative/antioxidative system is disrupted not only by the generally older age but also by the lower level of estrogen which believed to exhibit antioxidant effects (Sharma et al., 2015). In the current study, observation showed a significant decrease of TAC levels and BMD values in osteoporotic group compared to 
control group. These findings supported the results of previous reports by Sharma et al (2015) and Zhou et al (2016) who studied the effects of OS in the pathogenesis of PMO, and their results showed a decrease in total anti-oxidative status (TAS) in osteoporotic postmenopausal group. These findings supported the presence of oxidative damage in PMO.

Many studies suggested that ROS have a major role in postmenopausal osteoporosis. Dreher et al. 1998, claimed that missing or decreased expression of GPx could result in impaired osteoblast function and consecutive development of bone diseases such as osteoporosis. Also, Kobayashi et al (2009) indicated that the expression of RANKL increases with increased levels of oxidative stress, this means that increased oxidative stress stimulates the RANKL/RANK interaction. In this state, the formation, differentiation, and maturation of osteoclasts are promoted leading to the damage of bone tissue and development of osteoporosis.

There were controversial results and disparity among studies in the correlation between BMD and OS. The study of Sharma et al (2015) failed to detect a significant association between serum levels of OS-related biomarkers and BMD. One of an important results was that the correlation between TAC and BMD was found to be statistically significant in the osteoporotic group. This was consistent with previous study of Altindag et al (2008) who found a lower level of total anti-oxidative status (TAS), higher level of total oxidative status (TOS) and oxidative stress index (OSI) in PMW with osteoporosis. In addition, there was a significant negative correlation between OSI and BMD. Similarly, the study of Hahn et al (2008) who reported a significant correlation between BMD and antioxidant enzymes activity. Moreover, the studies of Lee et al (2015) and Han et al (2017) who observed that there was a significant positive correlation between BMD and serum levels of natural antioxidants. This literature controversy might be due to differences in ethnic populations, as well as other methodological techniques.

Taking together our data, indicated that, increased osteoclastic activity and decreased osteoblastic activity may be associated with an imbalance between oxidant and antioxidant status in postmenopausal osteoporosis. These results would suggest that OS might play a role in the development of PMO.

Thereby, our findings showed an association between increased serum levels of bone resorption marker OPN and reduced serum levels of TAC and decreased BMD values in postmenopausal osteoporosis women.

\section{Conclusion}

Our results support the published data on the role of Osteopontin in postmenopausal osteoporosis (PMO), that suggested OPN is a promising biomarker for identification of female individuals at high risk for osteoporosis. Therefore, it is recommended to use OPN as a complementary tool for assessing bone quality and for early prediction of fracture risk. The combined approach employing imaging techniques and biochemical markers of bone metabolism offer useful assistance in the evaluation 
and differential diagnosis of osteoporotic bone disease, it increases sensitivity and maintains specificity.

\section{REFERENCES}

Abdu Allah, A. M., El Tarhouny, S. A., Khodeer, S. A., \& Taha, I. M. (2017). Evaluation of osteopontn, c-telopeptide of type-I collagen and matrix metalloprotein in post- menopausal Egyptian women with osteoporosis. Biomedical Research (India), 28(8), 3645-3650.

Al-Nejjar, : Basma Ibraheem, Abdulrasheed, O. F., \& Al-shamaa, G. A. (2015). The Values of Osteopontin and Bone Specific Alkaline Phosphatase in Diagnosis of Osteoporosis. AComparative Study. Medical Journal of Babylon, 12(4), 987-998.

Altindag, O., Erel, O., Soran, N., Celik, H., \& Selek, S. (2008). Total oxidative/antioxidative status and relation to bone mineral density in osteoporosis. Rheumatology International, 28(4), 317-321.

Bergamini, C., Gambetti, S., Dondi, A., \& Cervellati, C. (2004). Oxygen, Reactive Oxygen Species and Tissue Damage. Current Pharmaceutical Design, 10(14), 1611-1626.

Beto, J. A. (2015). The Role of Calcium in Human Aging. Clinical Nutrition Research, $4(1), 1-8$.

Blake, G. M. (2010). The Clinical Role of Bone Density Scans in the Diagnosis and Treatment of Osteoporosis. In R. A. Adler (Ed.), Osteoporosis (pp. 77-95). Humana Press.

Boonen, S., \& Singer, A. J. (2008). Osteoporosis management: Impact of fracture type on cost and quality of life in patients at risk for fracture I. In Current Medical Research and Opinion. 24(6), 1781-1788.

Boskey, A. L., Maresca, M., Ullrich, W., Doty, S. B., Butler, W. T., \& Prince, C. W. (1993). Osteopontin-hydroxyapatite interactions in vitro: inhibition of hydroxyapatite formation and growth in a gelatin-gel. Bone and Mineral, 22(2), 147-159.

Chang, I.-C., Chiang, T.-I., Yeh, K.-T., Lee, H., \& Cheng, Y.-W. (2010). Increased serum osteopontin is a risk factor for osteoporosis in menopausal women. Osteoporosis International, 21(8), 1401-1409.

Cho, E.-H., Cho, K.-H., Lee, H. A., \& Kim, S.-W. (2013). High Serum Osteopontin Levels Are Associated with Low Bone Mineral Density in Postmenopausal Women. Journal of Korean Medical Science, 28(10), 1496-1499.

Delmas, P. D., Eastell, R., Garnero, P., Seibel, M. J., \& Stepan, J. (2000). The Use 
of Biochemical Markers of Bone Turnover in Osteoporosis. Osteoporosis International, 11(6), S2-S17.

Dreher, I., Schütze, N., Baur, A., Hesse, K., Schneider, D., Köhrle, J., \& Jakob, F. (1998). Selenoproteins are expressed in fetal human osteoblast-like cells. Biochemical and Biophysical Research Communications. 245(1): 101-107.

Fodor, D., Bondor, C., Albu, A., Simon, S., Craciun, A., \& Muntean, L. (2013). The Value of Osteopontin in the Assessment of Bone Mineral Density Status in Postmenopausal Women. Journal of Investigative Medicine, 61(1), 15-21.

Franzén, A., Hultenby, K., Reinholt, F. P., Önnerfjord, P., \& Heinegård, D. (2008). Altered osteoclast development and function in osteopontin deficient mice. Journal of Orthopaedic Research, 26(5), 721-728.

Golob, A. L., \& Laya, M. B. (2015). Osteoporosis. Medical Clinics of North America, 99(3), 587-606.

Gundberg, C. M. (2000). Biochemical Markers of Bone Formation. Clinics in Laboratory Medicine, 20(3), 489-502.

Hahn, M., Conterato, G. M. M., Frizzo, C. P., Augusti, P. R., da Silva, J. C. N., Unfer, T. C., \& Emanuelli, T. (2008). Effects of bone disease and calcium supplementation on antioxidant enzymes in postmenopausal women. Clinical Biochemistry, 41(1-2), 69-74.

Han, W., Bai, X., Wang, N., Han, L., Sun, X., \& Chen, X. (2017). Association between lumbar bone mineral density and serum uric acid in postmenopausal women: a cross-sectional study of healthy Chinese population. Archives of Osteoporosis, 12(1), 50(1-9).

Huang, W., Carlsen, B., Rudkin, G., Berry, M., Ishida, K., Yamaguchi, D. T., \& Miller, T. A. (2004). Osteopontin is a negative regulator of proliferation and differentiation in MC3T3-E1pre-osteoblastic cells. Bone, 34(5), 799-808.

Icer, M. A., \& Gezmen-Karadag, M. (2018). The multiple functions and mechanisms of osteopontin. In Clinical Biochemistry. 59(9): 17-27.

Kanis, J. A., \& Kanis, J. A. (1994). Assessment of fracture risk and its application to screening for postmenopausal osteoporosis: Synopsis of a WHO report. Osteoporosis International, 4(6), 368-381.

Kanis, J. A., McCloskey, E. V., Johansson, H., Cooper, C., Rizzoli, R., \& Reginster, J.-Y. (2013). European guidance for the diagnosis and management of osteoporosis in postmenopausal women. Osteoporosis International, 24(1), 2357.

Kling, J. M., Clarke, B. L., \& Sandhu, N. P. (2014). Osteoporosis Prevention, 
Screening, and Treatment: A Review. Journal of Women's Health, 23(7), 563572.

Kobayashi, Y., Udagawa, N., \& Takahashi, N. (2009). Action of RANKL and OPG for Osteoclastogenesis. Critical Reviews ${ }^{T M}$ in Eukaryotic Gene Expression, 19(1), 61-72.

Kwon, S., Wang, A. H., Sadowski, C. A., Yuksel, N., \& Doschak, M. R. (2018). Urinary Bone Turnover Markers as Target Indicators for Monitoring Bisphosphonate Drug Treatment in the Management of Osteoporosis. Current Drug Targets, 19(5), 451-459.

Lee, Y. J., Hong, J. Y., Kim, S. C., Joo, J. K., Na, Y. J., \& Lee, K. S. (2015). The association between oxidative stress and bone mineral density according to menopausal status of Korean women. Obstetrics \& Gynecology Science, 58(1), $46-52$.

Lorente Ramos, R. M., Azpeitia Armán, J., Arévalo Galeano, N., Muñoz Hernández, A., García Gómez, J. M., \& Gredilla Molinero, J. (2012). Absorciometría con rayos $\mathrm{X}$ de doble energía. Fundamentos, metodología y aplicaciones clínicas. Radiología, 54(5), 410-423.

McClung, M. R. (2005). The relationship between bone mineral density and fracture risk. Current Osteoporosis Reports, 3(2), 57-63.

Migliaccio, S., Greco, Fornari, Donini, \& Lenzi. (2011). Is obesity in women protective against osteoporosis? Diabetes, Metabolic Syndrome and Obesity: Targets and Therapy, 4, 273-282.

Seibel, M. J., \& Meier, C. (2010). Biochemical Markers of Bone Turnover - Basic Biochemistry and Variability. In R. A. Adler (Ed.), Osteoporosis (pp. 97-130). Humana Press.

Sharma, T., Islam, N., Ahmad, J., Akhtar, N., \& Beg, M. (2015). Correlation between bone mineral density and oxidative stress in postmenopausal women. Indian Journal of Endocrinology and Metabolism, 19(4), 491-479.

Shetty, S., Kapoor, N., Bondu, J., Thomas, N., \& Paul, T. (2016). Bone turnover markers: Emerging tool in the management of osteoporosis. Indian Journal of Endocrinology and Metabolism, 20(6), 846-852.

Syed, Z., \& Khan, A. (2002). Bone Densitometry: Applications and Limitations. Journal of Obstetrics and Gynaecology Canada, 24(6), 476-484.

Vasikaran, S., Eastell, R., Bruyère, O., Foldes, A. J., Garnero, P., Griesmacher, A., McClung, M., Morris, H. A., Silverman, S., Trenti, T., Wahl, D. A., Cooper, C., \& Kanis, J. A. (2011). Markers of bone turnover for the prediction of fracture risk and monitoring of osteoporosis treatment: a need for international 
reference standards. Osteoporosis International, 22(2), 391-420.

Veldurthy, V., Wei, R., Oz, L., Dhawan, P., Jeon, Y. H., \& Christakos, S. (2016). Vitamin D, calcium homeostasis and aging. Bone Research, 4(1), 16041.

Watts, N. B. (2004). Fundamentals and pitfalls of bone densitometry using dual-energy X-ray absorptiometry (DXA). Osteoporosis International, 15(11), 847-854.

Wei, Q., Huang, L., Tan, X., Chen, Z., Chen, S., \& Deng, W. (2016). Serum osteopontin levels in relation to bone mineral density and bone turnover markers in postmenopausal women. Scandinavian Journal of Clinical and Laboratory Investigation, 76(1), 33-39.

Zhou, Q., Zhu, L., Zhang, D., Li, N., Li, Q., Dai, P., Mao, Y., Li, X., Ma, J., \& Huang, S. (2016). Oxidative Stress-Related Biomarkers in Postmenopausal Osteoporosis: A Systematic Review and Meta-Analyses. Disease Markers, 2016, 1-12. 


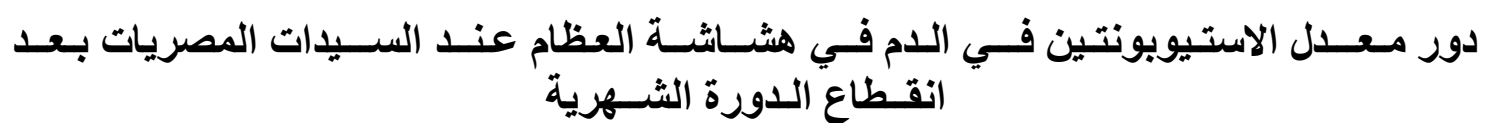

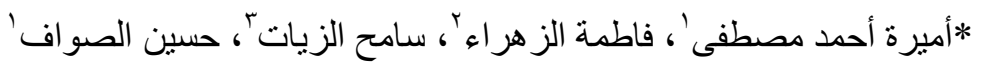

'قسم الكيمياء الحيوية كلية الصيدلة ــ الجامعة المصرية الروسية

r قسم الكيمياء الحيوية كلية الصيدلة (بنات) - جامعة الأزهر - القاهرة

"قنم الروماتزم والتأهيل كلية طب (بنين) - جامعة الأز هر - القاهرة

*Amira-ahmed@eru.edu.eg : البريد الاكتروني للباحث الرئيسي

الملخص العربي

مقدمة: النساء بعد سن اليأس أكثر عرضه للإصابة بهشاشنة العظام - حيث تصبح العظام ضعيفة، وسهلة الكسر - ويعتبر انخفاض معدل هرمون "الاستروجين" في مرحلة انقطاع الطمث من الإن الأسباب الرئيسية. الدراسات

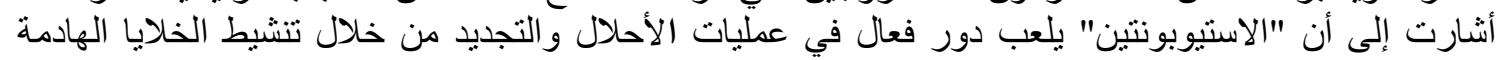

للعظام.

هدف البحث: يهدف هذا البحث إلى قياس معدلات تركيز "الاستيوبونتين" فى الدم بعد انقطاع الطمث ـ ـ وعمل

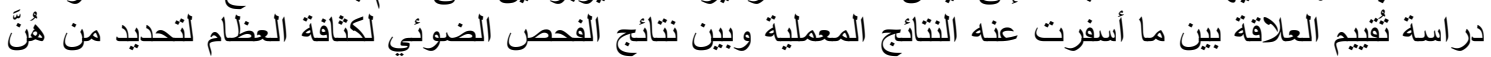
النساء الأكثر عرضه للإِصابة بكسور هثاشة العظام.

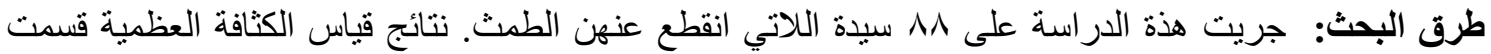

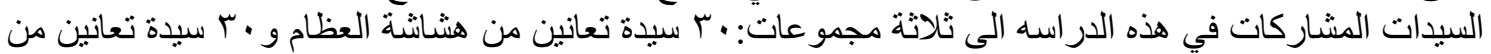

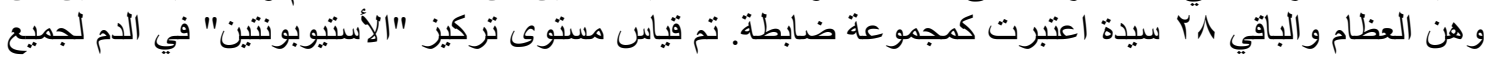

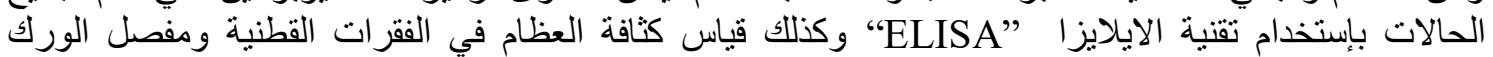

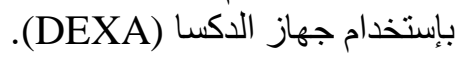

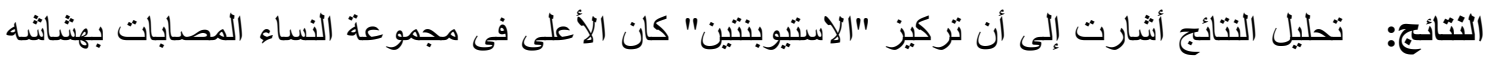

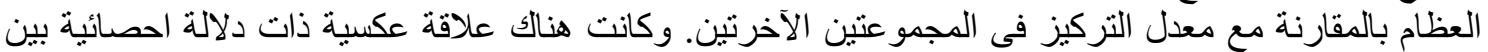

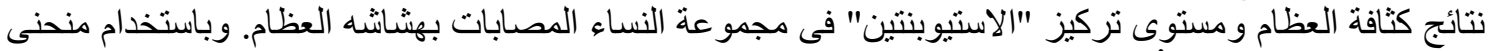

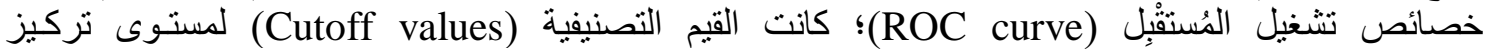

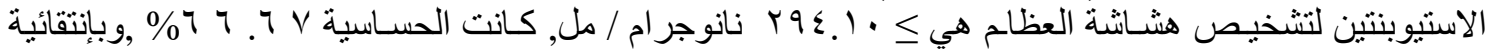

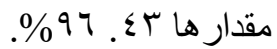

الاستتتاجات: أشارت نتائج هذه الدراسة إلى فاعلية مستوى تركيز "الاستيوبونتين" في الدم كمؤشر فعال و إيجابي

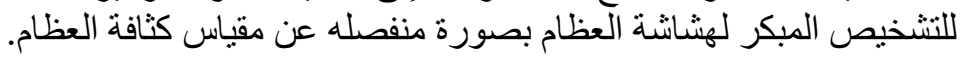
الكلمات المفتاحية: الأحلال و التجديد للنسيج العظمي؛ الاستروجين؛ الاستيوبنتين؛ هشاشة العظام. 